\title{
Assessment of Harmonic Distortion Sources in Power Networks with Capacitor Banks
}

\author{
Reyes S. Herrera, Patricio Salmerón, Salvador P. Litrán \\ ${ }^{1}$ Department of Electrical Engineering \\ E.T.S.I., Huelva University \\ Campus of La Rábida - Palos de la Frontera, 21819 Huelva (Spain) \\ Phone/Fax number:+0034 995 217572, e-mail: reyes.sanchez@die.uhu.es, patricio.uhu.es, salvador@uhu.es
}

\begin{abstract}
The identification of distortion sources in a power system is a topic unsolved. The problem has a difficult solution because there are elements in the system that do not produce harmonic but amplifies the existing in the electrical network. The most common of those elements is the capacitor, very used to compensate power factor at fundamental frequency. The capacitor behaviour makes that the indices proposed up now to identify distorsion indices fail in the presence of this element. In this paper, a new one is presented: the load characterization index. Besides using an extended equivalent circuit to represent the load, this index calculates the distortion introduced by the load, evaluating, besides the current distortion at its input, the voltage distortion at its terminals. The introduction of voltage assesing makes the index suitable to identify the linear and nonlinear loads in the power system even in the presence of capacitors.
\end{abstract}

\section{Key words}

Power Quality, Harmonic, Distortion Source, Distortion Assessment, Distribution Networks, Non-Linear Loads.

\section{Introduction}

In a liberalized electricity market, the electric power quality, EPQ, in the grid can be a critical competitive parameter and may represent a key to supply contracts. Both, measures and assessing consumers contribution to the EPQ are becoming increasingly important [1]-[8]. Harmonic distortion is one of the main aspects of power quality and it is the fastest growing in importance in recent years because of the proliferation of nonlinear and timevaring loads in power systems, which absorb nonsinusoidal currents.

In the thecnical papers there have been many proposals to establish the responsibility of each agent in the harmonic distortion of power distribution networks, [1][8]. One of the most relevant is the based on the harmonic active power sign, [2]-[3]. Other relevant group of proposal is the based on the current decomposition, [4]-[7].
All those procedures have their advantages and disadvantages. However, non of them are able to identify the true distortion sources on systems with the presence of capacitors, [8]-[11]. Indeed, it should be noted that while the loads that produce harmonics are undoubtedly non linear loads which introduce distortion in the system, other elements may have an important role in the propagation of harmonics in the networks, although they can not be listed as distortion sources. The capacitor is the most important one. It is widely used in power systems to compensate for low power factor of inductive loads. However, its performance is optimal only if the network operates in steady state sinusoidal conditions. In another case, the capacitor may cause serious problems, [8]-[11]. This is mainly due to its harmonic impedance, which decreases as frequency increases, and besides, it increases the distortion of the current that load consumes. In fact, on high harmonic order, capacitive impedances are really low and current is almost completely non active. So, for example, on high order of harmonic in presence of capacitor the determination of the active power sign is really difficult.

In this regard, a new procedure is presented in this paper that evaluates the distortion introduced by each load connected to a point of common connection, PCC. Besides, it takes into account the presence of capacitors to compensate the power factor at fundamental frequency and its behavior at high frequency. This procedure, which results in the so-called Load Characterization Index (LCI), could be implemented in commercial network analyzers to carry out the analysis of the responsibility of each load on the total distortion power system.

The behabiour of the index has been checked by simulation and experimental tests, based on industral applications where some problems have been detected. 


\section{The Load Characterization Index}

The problem presented in previous section makes necessary a new procedure which identifies linear loads in the presence of capacitors. In this section a new method is presented.

\section{A. Load Equivalente Circuit}

The background of the new method is within all those developments based on current decomposition into two parts. Thus, the analyzed load can be represented by two parallel branches: an impedance (a resistor $\mathrm{R}_{\mathrm{L} 1}$ in series to an inductance $\mathrm{L}_{1}$ ) and a nonlinear current source, $\mathbf{I}_{\mathrm{nl}}$. In [7], the linear part is represented by an inductive impedance as shown in figure 1 , calculated as the ratio between the voltage and current fundamental component. From that fundamental impedance, the corresponding to the rest of harmonic orders is calculated: the aproximation of considering that resistor remains the same with the frequency has been addopted; and on the other hand, inductance has been considered proportional to the harmonic order. The ratio of each order harmonic voltage and the corresponding impedance is that order linear current. The set of all the harmonics is the linear component of the current at the input of the load, $\mathbf{I}_{\text {lin }}$. The current nonlinear component, $\mathbf{I}_{\mathrm{n}}$, is calculated as the difference between the total, I, and the linear component, $\mathbf{I}_{\text {lin. }}$. The ratio of the rms value of nonlinear current and the rms value of the total current is called non linear index and it is assigned to the load by [7] to assess its responsibility in the total distortion of the system.

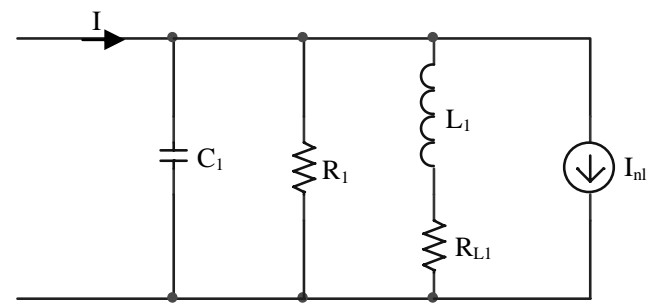

Fig. 1. Single-phase load equivalent circuit proposed in this paper

This procedure provides good results applyed to inductive loads. But, it does not identify linear loads in the presence of capacitor banks. To solve this problem, a new procedure is proposed in this paper. It goes on representing the load by an equivalent circuit with two parts: an impedance and a harmonic current source. However, in this case, the impedance is composed of three parallel branches: the first one is still an inductive impedance (a resistor $\mathrm{R}_{\mathrm{L} 1}$ in series to an inductance $L_{1}$ ), the second one is a resistance, $R_{1}$, and the third is a capacitor, $\mathrm{C}_{1}$, that represents the element of power factor compensation. Thus, the load is represented in the new procedure by the circuit shown in Figure 1. It takes into account, in addition to inductive loads, the pure capacitive or the inductive loads compensated by capacitors, besides the non-linear loads.

In this new procedure, the fundamental current is still flowing by the linear part of the load, as in the previous, but now, the determination of the value of each element in the linear part is not so easy as above. As there are three parallel branches, there are infinite possibilities of sharing fundamental current between them. This problem is solved in the procedure testing different values for each element. That is, the proposed index is calculated (as described below) for a specific number of circuits, varying the values of the elements of the linear part of the circuit that represents the actual load. In this way, an array of index values is obtained: an index value for each set of values considered for the elements.

The objective of the method is finding out the circuit more apropiate to the actual load. And the only constrain is that the current linear component flows throught the linear part of the equivalent circuit. Thus, as lower the index value, as better the tested circuit represents the actual load. Therefore, the circuit that provides the smallest value of the index will be considered as the representing to the studied load.

From now on, the methodology followed to fix the value of each element that constitutes the linear part of the load equivalent circuit is presented. From the voltage and current waveforms corresponding to the actual load, their frequency spectrum is determined. The ratio of current and voltage fundamental component provides the load admittance fundamental value, $Y_{1}=\mathbf{I}_{1} / \mathbf{V}_{1}$. $Y_{1}$ real part is the fundamental conductance, $G_{l}$, and its imaginary part is the fundamental susceptance, $\mathrm{B}_{\mathrm{l}}$. According to the susceptance sign, the load character is (negative susceptance correspondes to an inductive load and positive susceptance to a capacitive load). $\mathbf{V}_{1}, \mathbf{I}_{1}$ can be represented as shown in Figure 2a in the case of inductive load or as shown in Figure $2 \mathrm{~b}$ in case of capacitive load. This representation takes into account two of the load equivalent circuit branches: the inductive one and the capacitor. In fact, $\mathbf{V}_{1}$ represents the voltage fundamental component and $\mathbf{I}_{1}$ the current one. $\varphi_{1}$ represents the angle difference between voltage and current fundamental components, with current lags behind the voltage in the inductive case, Figure 2a, and leding in the capacitive one, Figure $2 \mathrm{~b}$. The presence of a capacitor is represented by a current, $\mathbf{I}_{\mathrm{C} 1}$, which leads $90^{\circ}$ the voltage fundamental component.

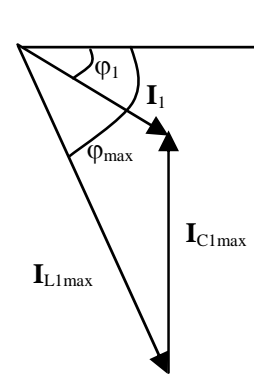

a) Inductive load

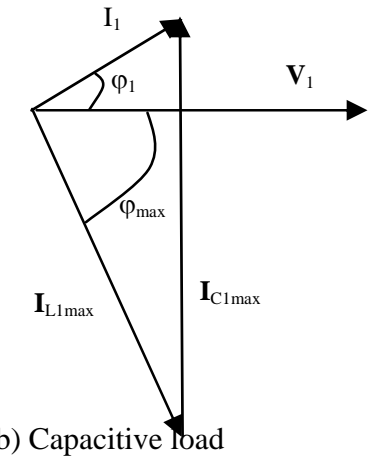

b) Capacitive road
Fig. 2. Vectorial diagram for calculating the load susceptance value

From Figure 2 diagram a minimum power factor is considered before compensation, which is given by $\varphi_{\max }$. Thus, the values of inductive branch elements and 
capacitor can be calculated in all the different possible cases considered. In one of them, the current flowing by the inductive branch is shown in the figure, $\mathbf{I}_{\mathrm{L} 1 \max }$, and its power factor is specified by $\cos \left(\varphi_{\max }\right)$. The current flowing by the capacitor in this case is specified by $\mathbf{I}_{\mathrm{C} 1 \mathrm{max}}$.

The current flowing by the inductive branch can be calculated from the current fundamental component and the minimum value of the power factor, $\cos \varphi_{\max }$, using the next expression:

$$
I_{L 1 \max }=\frac{I_{1} \cdot \cos \varphi_{1}}{\cos \varphi_{\max }}
$$

From this current, $\mathrm{I}_{\mathrm{C} 1 \mathrm{max}}$ can be calculated, depending on the load character. If the load is inductive:

$$
\begin{aligned}
& I_{C 1 \max }=I_{L 1 \max } \cdot \sin \varphi_{\max }-I_{1} \sin \varphi_{1}= \\
& =I_{1} \frac{\cos \varphi_{1}}{\cos \varphi_{\max }} \sin \varphi_{\max }-I_{1} \cdot \sin \varphi_{1}= \\
& =I_{1} \cos \varphi_{1} \cdot \tan \varphi_{\max }-I_{1} \cdot \sin \varphi_{1}
\end{aligned}
$$

In the same sense, if the load is capacitive:

$$
I_{C 1 \max }=I_{1} \cos \varphi_{1} \cdot \tan \varphi_{\max }+I_{1} \cdot \sin \varphi_{1}
$$

This value has been calculated from $\varphi_{\max }$ and the measured voltage and current fundamental components.

From the current flowing by the capacitor, given by (2)(3), the corresponding susceptance, $B_{1 \max }$, can be calculated as:

$$
B_{l \max }=\frac{I_{C 1 \max }}{V_{1}}
$$

This is the last case of the $\mathrm{m}$ considered in the procedure, and corresponds to the maximun value of capacitor susceptance $B_{1 \max }$. Its minimun value must be null in the case of an inductive load fully compensated. However in the case of a capacitive load, Figure 4b, the minimun value of the capacitor susceptance, $B_{\text {lmin }}$, is not zero, but it takes the following value:

$$
B_{l \min }=\frac{I_{1} \cdot \sin \left(\varphi_{1}\right)}{V_{1}}
$$

Therefore, $\mathrm{m}$ cases for capacitor admittance value are considered: at one end, the calculated in (4) and at the other end zero if the actual load is inductive or the value given in (5) if the actual load is capacitive. In the m-2 remaining cases, the susceptance value, $B_{1 i}$, will be considered a fraction of the range, calculated as the difference between maximum and minimum values, using the next expression:

$$
B_{l i}=B_{l \min }+\left(B_{l \max }-B_{l \min }\right) \frac{i-1}{m-1}
$$

where $\mathrm{i}$ is the number of test considered and $\mathrm{m}$ denotes the total number of values assigned to the susceptance. $B_{l i}$ is the assigned to the capacitive branch of circuit presented in figure 2 in the case $i, B_{1 i}=\omega C_{1 i}$.

With respect to the conductance value corresponding to the resistive branch of the load equivalent circuit shown in figure $1, G_{1}=1 / R_{1}$, two cases are considered: the null value corresponding to the non existence of the branch and the value of load total conductance $\mathrm{G}_{1}=\left(\mathrm{I}_{1} / \mathrm{V}_{1}\right) \cos \varphi_{1}$ corresponding to the case in which this element is the only responsible of the load active power requirement.

By comparing the $\mathrm{m}$ values for the susceptance of capacitor branch and the two values for the conductance of resistive branch, $2 \mathrm{~m}$ cases are possible to determine the values of the elements of actual load equivalent circuit, Figure 1.

In each analyzed case, i, the current flowing by capacitive and resistive branches is calculated as follows:

$$
\mathbf{I}_{1 G B i}=\mathbf{V}_{1} \cdot\left(G_{1 i}+j B_{1 i}\right)
$$

And so $\mathrm{Z}_{1 \mathrm{i}}$ value corresponding to the inductive branch impedance will be:

$$
\begin{aligned}
& Z_{1 i} \mid \varphi_{z i}=\frac{\mathbf{V}_{1}}{\mathbf{I}_{1}-\mathbf{I}_{1 G B i}} \\
& R_{1 i}=Z_{1 i} \cos \left(\varphi_{z i}\right) \\
& X_{1 i}=\omega L_{1 i}=Z_{1 i} \sin \left(\varphi_{z i}\right)
\end{aligned}
$$

where $R_{1 i}$ is the value of the resistence corresonding to the inductive branch in figure 2 and $\mathrm{L}_{1 \mathrm{i}}$ that of the inductance, both in case $\mathrm{i}$.

Until now the methodology for calculating the values of the load linear equivalent circuit elements at fundamental frequency has been presented. From them, their value at the harmonic orders considered can be calculated: the resistance value remains constant although the frequency changes, the inductance value is proportional to the frequency and so on the susceptance value. It is possible to calculate for each $2 \mathrm{~m}$ cases the current linear component flowing by the load. So, in case $\mathrm{i}$, the linear current, $\mathbf{I}_{\mathrm{li}}$, is the set of its harmonic components and it is given by the next expression:

$$
\mathbf{I}_{l i}=\sum_{j=1}^{n} \mathbf{V}_{j} \cdot G_{1 i}+\mathbf{V}_{j} \cdot Y_{1 i} \cdot j+\frac{\mathbf{V}_{j}}{R_{1 i}+X_{1 i} \cdot j}
$$

From the linear current flowing by the load in each case under consideration $\mathrm{i}, \mathbf{I}_{\mathrm{li}}$, the nonlinear current, $\mathbf{I}_{\mathrm{nli}}$, can be calculated as the difference between the total current flowing by the actual load and the linear current calculated in (9), as follows: 


$$
\mathbf{I}_{n l i}=\mathbf{I}-\mathbf{I}_{l i}
$$

And finally the modified nonlinear current index (NLC) is calculated as follows:

$$
N L C_{i}=\frac{\left\|\mathbf{I}_{n l i}\right\|}{\|\mathbf{I}\|}
$$

After calculating NLC corresponding to the $2 \mathrm{~m}$ cases considered, an array is obtained whose elements are those $2 \mathrm{~m}$ values. The minumun corresponds to the equivalent circuit that represents the load in the most exact way. Thus:

$$
N L C=\min \left(N L C_{i}\right)
$$

This modified non-linear current index identifies all linear inductive loads, achiving the assingment to them a null responsability in the system distortion. As an illustration of this fact, a power system is presented, shown in Figure 3, that comprises a PCC that feeds three loads:

- Load 1: A three-phase linear load consisting of an inductance in parallel to a resistor.

- Load 2: A non-linear load consisting of three ac singlephase regulators with an inductive load (an inductor in series to a resistor) connected in star.

- Load 3: A three-phase rectifier with a capacitive impedance (a capacitor in parallel with a resistor) on dc side.

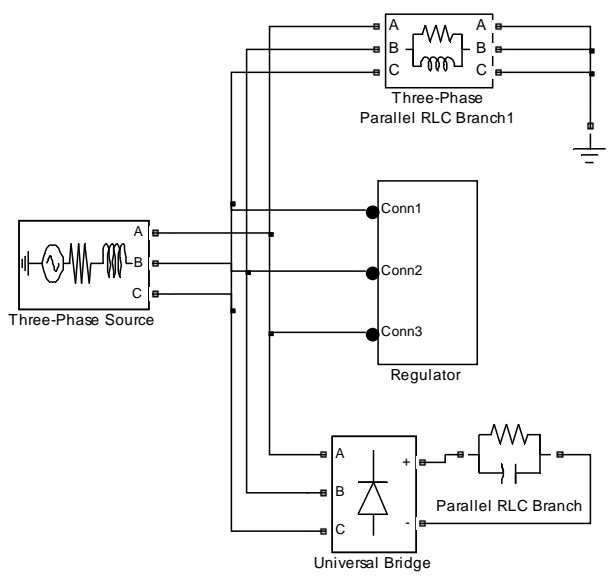

Fig. 3 Simulation Platform to test the NLC

In this system, the NL of [7] calculated from the voltage at the PCC and the current flowing by load 1, has a nonzero value $(8.28 \%)$, whereas the modified index provides a null value, which identifies the load as linear. For load 2, both indices results are $34.85 \%$ and for load 3 , $43.08 \%$ and $30.0 \%$, respectivelly.

Therefore, the index presented in [7] does not identify as linear the inductive impedances composed of a resistor in parallel to an inductance. Nevertheless, the modified nonlinear current index identifies the inductive linear loads with any configuration. And so, no responsability in the global distortion of the system is assigned to them.

\section{B. An index based on voltage distortion}

Thus, the modified non-linear current index improves the results of the earlier, presented in [7]. Among other, this index extends the linear load circuit used to represent the linear part of the actual load. It allows the representation of not only inductive loads but capacitive. However, applying this index to a capacitive linear load, the value obtained is not null.

Therefore, to identify linear loads with capacitors, it must also be taken into account one crucial fact: the capacitors do not generate harmonics, but amplify the present in the system. In fact, analogous to the behavior of the inductances, which amplify voltage harmonics but not those of current, the capacitors amplify current harmonics but not those of voltage, [5]. It is not therefore possible to identify a linear load in the presence of capacitors using an index based on current, as most of the proposals published so far. Indeed, the index used to identify as linear the linear loads with capacitor can not be based on current assesment, but could be based on voltage. And it should be carried out without losing the linear model discussed above. Therefore, the use of the model shown in Figure 4 is proposed in this paper. The impedance connected in series to the voltage source is exactly the same as the calculated above to connect in parallel to the current source. Thus, Figure 4 could represent the Thevenin model associated to the Norton model presented in Figure 1.

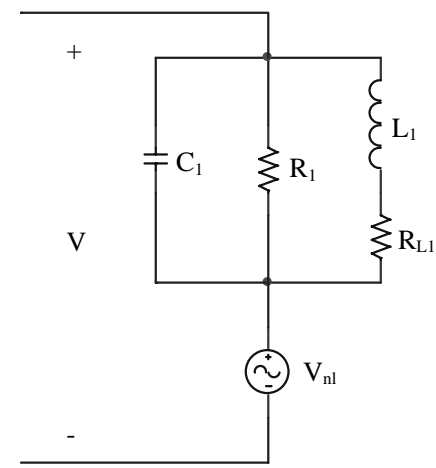

Fig. 4. Single-phase load equivalent circuit in procedure based on voltage

The index keeps the values obtained for the elements of the equivalent circuit, figures 1 and 4 . From them, the index calculates in each case i the linear voltage drop in the load, $\mathbf{V}_{\mathrm{li}}$, that is:

$$
\mathbf{V}_{l i}=\sum_{j=1}^{n} \frac{\mathbf{I}_{1}}{G_{1 i}+Y_{1 i} \cdot j+\frac{1}{R_{1 i}+X_{1 i} \cdot j}}
$$

From this linear voltage of case $\mathrm{i}, \mathbf{V}_{\mathrm{li}}$, the nonlinear component of voltage drops in the load, $\mathbf{V}_{\text {nli }}$, can be calculated:

$$
\mathbf{V}_{n l i}=\mathbf{V}-\mathbf{V}_{l i}
$$


And finally the nonlinear voltage index (NLV) is defined as follows:

$$
N L V_{i}=\frac{\left\|\mathbf{V}_{n l i}\right\|}{\|\mathbf{V}\|}
$$

So, now there are two arrays of results: one with $2 \mathrm{~m}$ NLC and another with $2 \mathrm{~m}$ NLV. The first array minimum value corresponds to the Norton model that best fits the analyzed load and the second array minimum value corresponds to the Thevenin model that best fits the analyzed load. And now, the load charactization index, LCI, may be defined as follows: if any of these two minima is close to 0 , the load is linear and the load characterizing index is null. Otherwise, the load is a distortion source and the LCI value is the corresponding to NLC. In fact, the Thevenin circuit has been useful to identify the linear loads with any configuration. Nevertheless, as the distortion on voltage use to be less than that on current, the responsibility on the system distortion fixed by means of NLC may be the most suitable option and LCI value will be as follows:

$$
L C I=\left\{\begin{array}{l}
\text { when } \quad N L C \square 0, \quad L C I=0 \\
\text { when } N L V \square 0, \quad L C I=0 \\
\text { otherwise, } \quad L C I=N L C
\end{array}\right.
$$

Thus, LCI value will be the necessary tool to attribute to each nonlinear load its responsibility in the power system total distortion.

\section{Sumulation and Experimental Results}

To check the LCI validity, the system studied in [11] has been simulated, Figure 5. It is a simple but crucial case that illustrates the real practical operating conditions. It consits of a voltage source supplying two loads: the first one is a non-linear load with a high power rate simulated as a rectifier with a capacitive impedance in dc side, and the second one is a linear load with a much smaller power rate simulated as an inductive impedance in parallel to a capacitor, Figure 5. On the other hand, the indices evaluated has been: Global Harmonic Index, GHI, based on harmonic active power sign, [2]-[3]; nonlinear index NL, as presented in [7]; and LCI, presented in this paper. They have been calculated at the input of each load and in the PCC. The phase one of the balance three-phase voltage in PCC is shown in Figure 6a. The phase one of the current at the input of the non-linear load is shown in Figure 6b and that at the input of the linear load in Figure 6c. Figure $6 \mathrm{~b}$ shows a non sinusoidal waveform obtained, for example in arc furnaces. Its distortion makes, through the source impedance, that the voltage in PCC is non sinusoidal, too, Figure 6a. Moreover, Figure 6c shows a drastically non sinusoidal current at the input of a linear load. It illustrates the main problem of the distortion sources identification: the particular behaviour of capacitor when they are connected to a distorted PCC. It is a linear load but the current at its input presents a higher distortion than the present in the power system.
In table I, the indices values calculated are presented. It can be seen that GHI and NL assess responsability to the linear load for the distortion in the system. Morover, NL establishes a higher responsability to the linear load than the assigned to the non-linear load. However, LCI identifies the linear load as linear specifing the non-linear load as the only distorsion source in the system. Besides, on the other hand, it must be indicated that the new index can be calculated from the only measures taken by a comercial analizer.

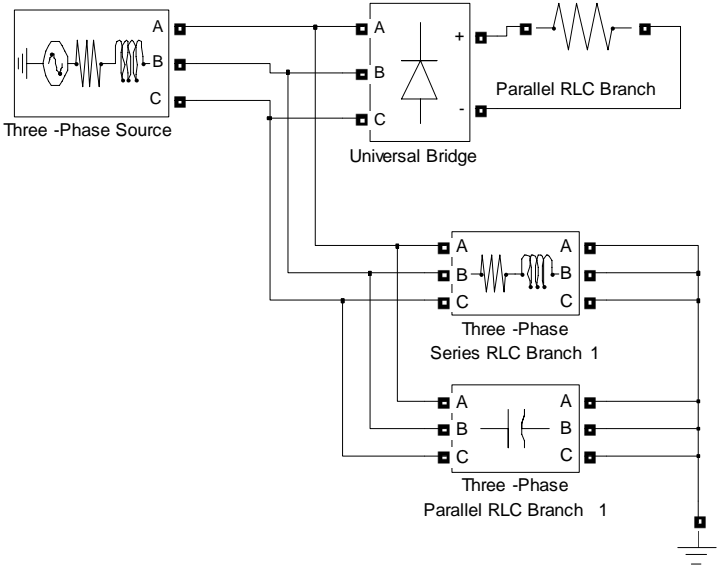

Fig. 5. Simulation and Experimental Platform

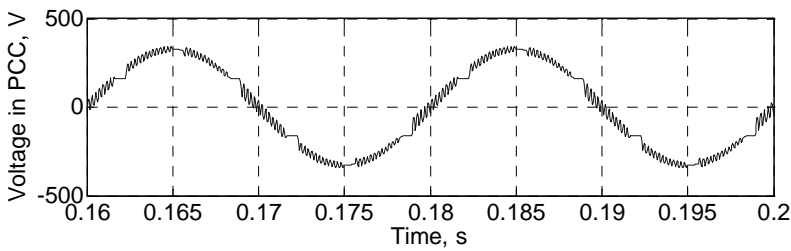

a) Voltage on PCC

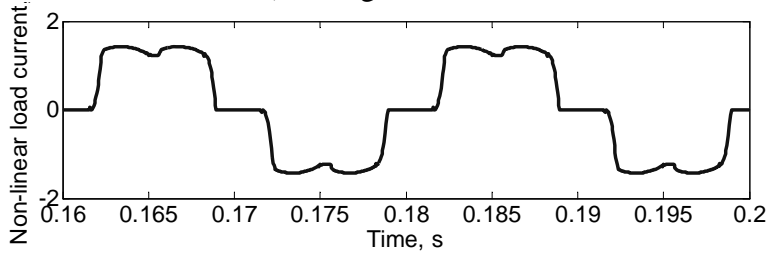

b) Current at non-linear load input

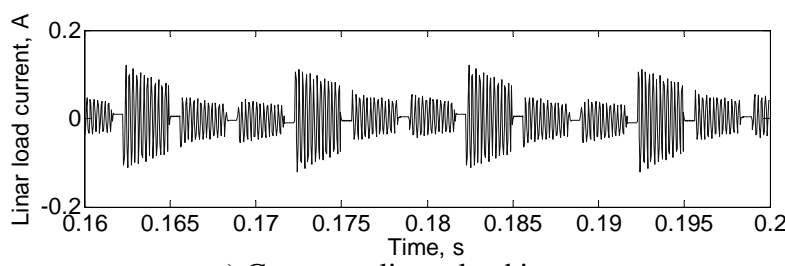

c) Current at linear load input

Figure 6. Phase one of magnitude obtained in the simulation system

Table I. Simulation results

\begin{tabular}{|l|c|c|c|}
\hline & PCC & Non-linear load & Linear load \\
\hline GHI & 27,52 & 27,17 & 5,00 \\
\hline NL & 28,22 & 27,81 & 99,69 \\
\hline LCI & 27,92 & 27,62 & 0,00 \\
\hline
\end{tabular}

Finally, an experimental platform has been designed to represent the analized power system. It is constituted by a 
voltage source supplying two loads: a rectifier with a capacitive impedance in dc side and a linear load composed of an inductive impedance in parallel to a capacitor. The configuration is therefore similar to the presented in Figure 5 althoug in the experimental platfom the power rates of both loads are more similar than those in simulation system (one is double to the other).

Voltage and current have been measured with a data acquisition cards compatible with Matlab-Simulink and developed by dSPACE. Real-Time Interface (RTI) from dSPACE together with Real Time Workshop (RTW) from Mathworks automatically generate real time code. It allows the processor board to be programmed and $\mathrm{I} / \mathrm{O}$ boards to be selected.

The phase one of the voltage in PCC is shown in Figure 7a. It is a nonsinusoidal voltage due to the non sinusoidal current at the input of the first load and the source impedance. Figure $7 \mathrm{~b}$ shows the phase one of the current at the input of the first load and Figure $7 c$ that at the input of the second load. Figure 7c shows a non sinusoidal current, although its distortion is much smaller than the corresponding to the simulation system due to the fact that now the current at the input of non linear load is only the double of that at the input of linear load.

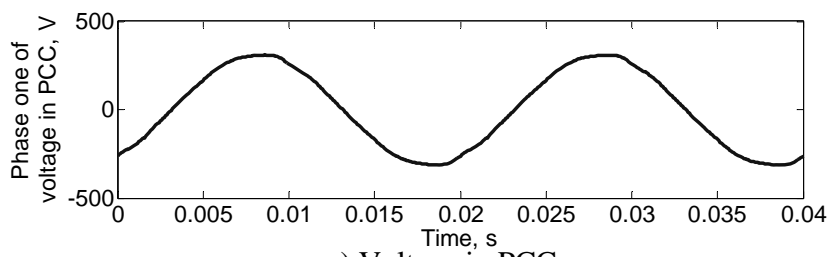

a) Voltage in PCC

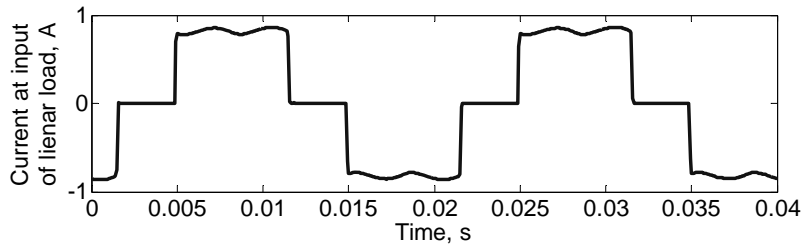

b) Current at the input of non linear load

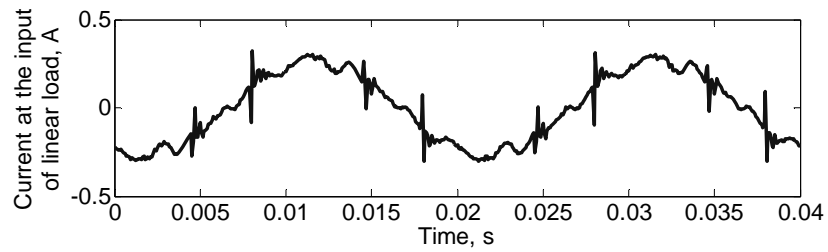

c) Current at the input of linear load

Figure 7. Phase one of magnitude obtained in experimental system

Table II. Experimental results

\begin{tabular}{|l|c|c|}
\hline & Non-linear load & Linear load \\
\hline GHI & 19,12 & 8,23 \\
\hline NL & 29,08 & 20,49 \\
\hline LCI & 28,64 & 0,00 \\
\hline
\end{tabular}

In table II, the values of the indices calculated fron voltage and current obtained in experimental system are presented. It can be seen that all of them make responsible for system distortion to the non linear load and the values goes from the 19,12 obtained by GHI and the 29,08 by NL.
LCI has obtained 28,64, very closed to NL. With respect to the linear load, all the indices make it responsible for system distortion except LCI, which identifies it as linear and makes the non-linear load the only responsible for system distortion.

\section{Conclusion}

In this paper the main problem within the identification of distortion sources in a power system is analized: the presence of capacitors. The problem has been identified and studied in several papers in the Literature. In particular, it has been shown that the indices proposed up now fail in the presence of capacitors because they specify linear loads as distortion sources. In this paper the load characterization index (LCI) is presented. It is able to distinguish the linear and non-linear loads. Thus, the non-linear are considered the only responsible for the distortion present at the point of common connection. This index has been applied to simulation and experimental systems. The results obtained in both, confirm the validity of the new index proposed in this paper in the presence of capacitors.

\section{References}

[1] P. Salmerón, F. J. Alcántara, A New Technique for Unbalance Current and Voltage Estimation With Neural Networks, IEEE Transactions on Power Systems, Vol. 20, No. 2, May 2005, pp. 852-858.

[2] C. Muscas, Assessment of Electrical Power Quality: Indices for Identifying Disturbing Loads, ETEP, Vol. 8, No. 4, July/August 1998.

[3] J.E. Farach, W.M. Grady y A. Arapostathis. An Optimal Procedure for Placing Sensors and Estimating the Locations of Harmonic Sources in Power Systems. IEEE Transations on Power Delivery, Vol. 8, No. 3, July 1993.

[4] L.S. Czarnecki, Distortion Power in Systems with Nonsinusoidal Voltage, Proc. IEE, Vol. 139, Pt. B, No. 3, pp. 276-280, May 1992.

[5] Srinivasan, K. On Separating Customer and Supply Side Harmonic Contributions, IEEE Trans. on Power Delivery, vol. 11, No. 2, April 1996.

[6] K. Srinivasan, R. Jutras. Conformin and non-Conforming Current for Atributting Steady State Power Quality Problems. IEEE Transactions on Power Delivery, Vol. 13, No. 1, January 1998.

[7] A. Dell'Aquila, M. Marinelli, V. G. Monopoli y P. Zanchetta. New Power-Quality Assessment Criteria for Supply Under Unbalanced and Nonsinusoidal Conditions. IEEE Transations on Power Delivery, Vol. 19, No. 3, July 2004.

[8] C. Muscas, L. Peretto, S. Sulis, R. Tinarelli. Investigation on Multipoint Measurement Techniques for PQ Monitoring. IEEE Transaction on Instrumentation and Measurement, Vol 55, No. 5, Oct. 2006.

[9] P. Salmerón, S.P. Litrán, J.R. Vázquez, R.S. Herrera. Análisis de Distintos Métodos para Identificar las Fuentes de Distorsión en Redes Eléctricas. XVIII Reunión de Grupos de Investigación de Ingeniería Eléctrica, 13-14 de marzo de 2008, Santander, España.

[10] L.I. Eguiluz, J.C. Lavandero, M. Odriozola, V.M. López. Methodology for Harmonic Distortion Level Determination. International Conference on Renowable Energy and Power Quality, March 2008, Santander, Spain.

[11] N. Locci, C. Muscas, S. Sulis. On the Measurements on Power Quality Indexes for Harmonic Distortion in the Presence of Capacitors. IEEE Transaction on Instrumentation and Measurement, Vol 56, No. 5, Oct. 2007. 